Rabaska

Revue d'ethnologie de l'Amérique française

\title{
La Société historique de Saint-Boniface
}

\section{Gilles Lesage}

Volume 2, 2004

URI : https://id.erudit.org/iderudit/201703ar

DOI : https://doi.org/10.7202/201703ar

Aller au sommaire du numéro

Éditeur(s)

Société québécoise d'ethnologie

ISSN

1703-7433 (imprimé)

1916-7350 (numérique)

Découvrir la revue

Citer ce document

Lesage, G. (2004). La Société historique de Saint-Boniface. Rabaska, 2, 321-324.

https://doi.org/10.7202/201703ar

Ce document est protégé par la loi sur le droit d'auteur. L'utilisation des services d’Érudit (y compris la reproduction) est assujettie à sa politique d'utilisation que vous pouvez consulter en ligne.

https://apropos.erudit.org/fr/usagers/politique-dutilisation/
Cet article est diffusé et préservé par Érudit.

Érudit est un consortium interuniversitaire sans but lucratif composé de l’Université de Montréal, l'Université Laval et l'Université du Québec à Montréal. Il a pour mission la promotion et la valorisation de la recherche. https://www.erudit.org/fr/ 
OuEsT

\section{La Société historique de Saint-Boniface}

Centre du patrimoine

340, boulevard Provencher

Saint-Boniface (MB) R2H 0G7
Téléphone : (204) 233-4888

Télécopieur : (204) 231-2562

Courriel : shsb@shsb.mb.ca

Toile : www.shsb.mb.ca

\section{Origine et mandat}

La Société historique de Saint-Boniface (SHSB) a été fondée en 1902. Depuis ce jour, elle poursuit son mandat de conserver et de promouvoir le patrimoine fruit de la présence des francophones dans l'Ouest canadien et en particulier au Manitoba. La SHSB est active dans tous les domaines du patrimoine métis et francophone du Manitoba et de l'Ouest canadien : recherches historiques, muséologie, généalogie, archives, publication, conférences, expositions, etc. Avec l'ouverture du Centre du patrimoine, la SHSB œuvre maintenant dans trois secteurs clé : le service d'archives, le service de la bibliothèque historique et le service de généalogie. Sont aussi associées à ces services les activités de recherche, de consultation et de promotion. Dans ce but, la SHSB publie un bulletin, organise des colloques et des conférences, gère le Lieu historique national du Canada de la Maison-Riel et répond à de nombreuses demandes de recherches. Collaborant avec les Éditions du Blé, la SHSB a publié plusieurs titres dans la collection des « Cahiers d'histoire de la Société historique de Saint-Boniface ».

\section{Service de la bibliothèque}

En plus d'une bibliothèque de référence de près de 8000 titres, la SHSB a aussi fait l'acquisition de la Bibliothèque nationale de l'Archevêché de SaintBoniface, une des plus importantes collections de livres (environ 30000 titres) et de brochures portant, entre autres, sur l'histoire des francophones de l'Ouest. Plusieurs documents portent sur la question des écoles. Il y aussi un certain nombre de documents au sujet des caisses populaires et de l'Action catholique. Plusieurs périodiques et journaux font partie de cette collection, dont Le Manitoba et Le Métis.

\section{La Maison-Riel}

La SHSB gère le Lieu historique national du Canada de la Maison-Riel depuis 1980. Ce contrat de gestion avec le gouvernement fédéral a permis une plus grande spécialisation dans les domaines de l'interprétation, de la 
programmation, des activités de musées et de la formation de guides. Il permet aussi de faire la promotion du patrimoine métis auprès du grand public et des écoles. Le Lieu historique national de la Maison-Riel, situé au 330, chemin River, est ouvert au public durant l'été (de la longue fin de semaine de mai jusqu'à la longue fin de semaine de septembre) de $10 \mathrm{~h}$ à $18 \mathrm{~h}$, sept jours sur sept. Renseignements : (204) 257-1783

\section{Service de généalogie}

La SHSB, appuyée de plusieurs bénévoles, assure un service de généalogie mettant à la disposition du public une riche documentation et un généalogiste expérimenté. Ces ressources et ce service portent sur les familles métisses et francophones de l'Ouest canadien. La SHSB assure et favorise la publication de matériel généalogique, offre des ateliers en techniques de recherches et répond à de multiples demandes de renseignements. Les ressources comprennent des microfilms (Fichier Rivest, Henderson Directories, Scrips (Scripts : certificats) Métis, Recensement, Journaux, Registres de paroisses du Manitoba et du Québec, des Greffes de notaires), des microfiches (Fichier Loiselle), des fonds d'archives (Picton), des dictionnaires (Drouin, Langlois, Tanguay, Jetté, White), des répertoires de mariages de nombreuses paroisses du Québec et du Manitoba, des bibliographies (Mennie de Varennes), des histoires de paroisses et de municipalités, des histoires de familles et des histoires locales, sans compter les bases de données (PRDH, recensements, registres sacramentaux). On y trouve aussi une abondante documentation rassemblée en grande partie par Alfred Fortier par dossiers de personnes ou de familles. Plusieurs bases de données donnent accès aux relevés de registres de paroisse du Manitoba, de la Saskatchewan et des États-Unis, aux scrips (scripts : certificats) des Métis, aux contrats de voyageurs, à certains recensements, aux photographies et à la bibliothèque.

\section{Service des archives}

Depuis plus de 100 ans, la Société historique s'intéresse à l'évolution sociale, culturelle, politique, juridique et religieuse de la population d'expression française au Manitoba. Elle acquiert documents textuels et iconographiques, cartes, photographies, livres, journaux, enregistrements et films qui constituent les archives. Elle fait le traitement des archives, en facilite l'accès et en assure la préservation. L'accès en ligne de plusieurs descriptions de fonds d'archives et de documents numérisés se trouve au site www.shsb.mb.ca. Parmi les fonds d'archives déposés à la SHSB, les fonds de Louis Riel, de la Société historique métisse, de la Corporation archiépiscopale catholique romaine de Saint-Boniface et de Joseph-Noël Ritchot sont particulièrement prisés. Ensemble ils contiennent la deuxième plus riche collection de docu- 
ments originaux de Louis Riel. S'y ajoutent les archives de la radio et de la télévision de la Société Radio-Canada au Manitoba, du Cercle Molière, de l'Association d'éducation des Canadiens-Français du Manitoba, de la Société franco-manitobaine et de bien d'autres qui documentent tous les aspects de la vie passée et présente des collectivités francophones et des Métis du Manitoba. La collection comprend aussi des photographies, des images animées, des cartes et des affiches. De fait, la SHSB est la seule institution provinciale vouée à l'acquisition des archives du Manitoba français et métis.

Plusieurs fonds d'archives documentent l'ethnologie et le folklore du Manitoba français et métis. L'un des plus connus est le fonds Henri Létourneau. Chroniqueur, folkloriste et conservateur, Henri Létourneau est né en septembre 1907 à Sainte-Delphine, Saskatchewan. Il est fils de DominaAlexandre et de Marie-Alvina Chouinard. Quittant l'école à quatorze ans, Henri Létourneau travailla à plusieurs endroits dans les provinces de l'Ouest. Il s'établit ensuite à Saint-Boniface épousant Rose Leclerc. À partir des années 1950 , il prit davantage intérêt au patrimoine métis et canadien-français commençant à recueillir des chants et des légendes du folklore métis et canadien-français. Dans les années 1960, il collectionna des objets métis et contribua largement au développement de la collection d'artefacts métis du Musée de Saint-Boniface où il devint conservateur de 1968 à sa retraite en 1985. Durant les années 1970, il entreprit un projet d'enregistrement du folklore métis pour le Musée National de l'Homme (aujourd'hui Musée canadien des civilisations), Ottawa. Plus tard, la Fondation C.K.S.B. lui octroya une subvention pour faire la transcription des bandes sonores. En 1978, il publia Henri Létourneau raconte aux Éditions Bois-Brûlés suivi d'une édition revisée en 1980. Ce fonds documente le folklore métis et canadien-français du Manitoba et de la Saskatchewan. On y trouve des légendes, de nombreuses chansons, de la musique, des descriptions de jeux d'enfants, des recettes, des témoignages, des récits d'événements du passé, des descriptions de métiers et des renseignements biographiques grâce à des interviews avec des Métis et Canadiens français de Saint-François-Xavier, Baie-Saint-Paul, Piapot, Duck-Lake, Batoche, Portage La Prairie, Saint-Boniface, Richer, Skull-Creek, Eastend, Saint-Labre, Woodridge, Rosthern, Sainte-Rose-du-Lac, Cayer, Saint-Norbert, Belcourt (Dakota du Nord), Saint-Eustache, Saint-Lazare, Biscarth, Saint-Joseph, San-Clara, Saint-Georges, Saint-Pierre, Saint-Vital, Saint-Charles, Saint-Malo, Otterburne, Saint-Laurent et Sainte-Anne-desChênes.

Un autre fonds déposé aux archives de la SHSB est celui de Monique Hébert qui documente plus particulièrement les femmes dans le monde de l'enseignement. En 1994, Monique Hébert recevait son doctorat en histoire de l'Université du Manitoba ayant complété sa thèse sur la contribution des 
Franco-Manitobaines à la survie de la communauté de 1916 à 1947. Au cours de ses recherches elle a assuré un projet d'histoire orale avec la Société historique de Saint-Boniface sur le rôle des Franco-Manitobaines dans la survie de la communauté. De retour au Québec, elle enseigna à l'Université d'Ottawa et à l'Université du Québec à Hull. Ce fonds comprend des documents produits par Monique Hébert au cours de ses recherches portant sur les femmes franco-manitobaines. Les documents ainsi produits comprennent des notes de recherche, des photocopies de documents des archives de l'Association d'éducation des Canadiens-Français du Manitoba (dont des copies des rapports de visiteur et de la correspondance); de la correspondance au sujet de la thèse (avec sa directrice) ; des compilations de données ; d'autres documents de recherche (en large partie des photocopies d'articles) et des cassettes sonores d'entrevues avec des femmes franco-manitobaines (y compris des sommaires et les ententes de dépôt et d'accès).

Un autre projet d'histoire orale a été réalisé par Ken Sylvester dans le cadre d'une recherche en vue d'une thèse de doctorat. Le projet a été nommé Montcalm Oral History Project et les entrevues ont été faites en anglais. Des entrevues ont été faites avec les personnes suivantes: Jean-Louis Perron ; Joseph Saurette ; Bernard Ayotte ; Ben Rémillard ; Aimé Barnabé ; Bernard Lafond ; Annette Sabourin ; Pierre Baril ; Arthur Devigne ; Irene Milne ; Ian Milne ; Leslie Forrester ; Odile Parent. Les interviews, selon le mode biographique, traitent de personnes ayant vécu à Saint-Joseph, Letellier, SaintPierre, Marais, Saint-Jean-Baptiste, Oak-Farm. Ces enregistrements sonores sont aussi déposés aux archives de la SHSB.

Ainsi, d'autres fonds d'archives déposés à la Société historique de SaintBoniface, tant d'organismes que d'individus documentent de façon plus particulière l'ethnographie et la vie folklorique du Manitoba français et métis. Parmi ceux-ci mentionnons celui de Marcien Ferland et de l'Ensemble folklorique de la Rivière-Rouge. Ces fonds d'archives peuvent être consultés grâce à des bases de données accessibles à l'adresse suivante : www.shsb.mb.ca/dbtw-wpd/textbase/webmenu1.htm

Gilles Lesage 